\title{
Tablet-Administered Screening Tests for the Detection of Major and Mild Cognitive Disorders - Preliminary Findings of a Comparative Study
}

\author{
https://doi.org/10.3991/ijim.v14i11.14629 \\ Andreas Solias $\left.{ }^{(}\right)$, Agisilaos Chaldogeridis, Areti Batzikosta, \\ Magdalini Tsolaki \\ Aristotle University of Thessaloniki, Thessaloniki, Greece \\ asolias@med.uoa.gr
}

\begin{abstract}
Preventative screening of at-risk population is a crucial step in the early detection of dementia. Building alternative and ecologically validated screening tests are challenging. This pilot project attempts a) to assess the effectiveness of a culturally oriented computerized screening test for major neurocognitive disorder (eHAST) b) to compare it with the Greek version of the UCSF Brain Health Assessment (BHA) which is culturally neutral. Our study focused in a) evaluation of eHAST's discriminant ability between groups of older adults with cognitive concerns (OACC) $(n=35)$ vs older adults with Mild Cognitive Impairment $(\mathrm{MCI})(\mathrm{n}=114)$ and dementia $(\mathrm{n}=67)$, and MCI vs dementia in a sample of 216 cases. b) comparison of eHAST's discriminant ability vs BHA's in 116 subjects. The absence of normative data for both of the tests led to balance the sample regarding age and educational level for the comparisons between groups of OACC vs MCI $(n=56)$ and MCI vs dementia cases $(n=60)$. All participants were neuropsychologically assessed, clinically diagnosed. Results showed a) Reliability check (Cronbach's alpha) of eHAST was 0.9 and discriminant analyses in separating groups of OACC vs MCI displayed a correct classification rate of $77.3 \%$, for MCI vs dementia $80.6 \%$ and OACC vs dementia $89.1 \%$ (cross-validated). b) In OACC vs MCI comparison, BHA's discriminant ability was $67.3 \%$ and eHAST's was $60.7 \%$. In MCI vs dementia comparison, BHA's discriminant ability was $77.2 \%$ and eHAST's was $76.7 \%$ (cross-validated). These preliminary results of the implementation of eHAST are encouraging as the test presents sufficient discriminant ability between groups of OACC - MCI, MCI - Dementia \& OACC - Dementia. Also, performs similar scores to BHA, which is a state-ofthe-art screening test. The use of a tablet pc in the assessment of cognitive disorders in the elderly can have a catalytic role in the detection of dementia.
\end{abstract}

Keywords - Computer-based screening tests, cognitive disorders, Alzheimer's 


\section{Introduction}

\subsection{Theoretical background}

Dementia is evolving into a major public health crisis across the globe. Recent prospective studies paint a very bleak picture of the dementia landscape [1], [2], [3]. Advances in modern medicine allow for the diagnosis of Mild Cognitive Impairment (MCI) and Dementia as well as the identification of the disorder's subtype and probable underlying pathology, in specialized settings such as memory clinics. At the same time, a large percentage of the global population lacks access to such services and a large percentage of patients (over 50\%) who are examined in primary health care services remain undiagnosed [4], [5], [6], [7]. Moreover, diagnosis takes place late for a large number of patients, often occurring at a relatively advanced or even at a very severe stage of dementia [8], [9]. It is especially concerning that such findings come from countries such as the U.S.A. and the U.K. who have a large number of general practitioners, specialists and a lot of experience in dementia care.

Focusing in low-income countries, where the number of older adults is expected to quadruple between 2015 - 2050, (239\% increase versus 56\% increase in high-income countries), the corresponding number of dementia patients is expected to be double [2]. Part of the limited budget for health services in low-income countries has to be allocated to dementia, while these services are dealing at the same time with child mortality, infectious diseases and other urgent issues.

Screening the population for dementia is the first decisive step for early and effective treatment of the disease, despite any disagreements regarding which segment of the population should be screened [4], [5], [10]. In general, country-wide screening for dementia (in the same way as for diabetes and hypertension) is not advised as it has not proven to be cost-effective [11]. At the same time, the evaluation of cognitive functions in the elderly has many supporters. Researchers have expressed the will to create valid, self-administered tests especially for community screening [12], [13]. Self-administration could negate the issue of cost up to a point.

Early dementia diagnosis provides the opportunity to better utilize the benefits of the combination of available drugs and non-pharmaceutical interventions. At the earliest stage of the disease, the patients can ask for treatment by themselves and also manage personal, family and property matters. An effort is being made to detect cognitive impairment at the MCI stage [14], [15], [16], which is often a precursor to dementia. Annual transition of MCI patients to dementia is calculated at 2-31\% [17], [18]. It is commonly accepted that a diagnosis of MCI increases the risk of progression to dementia [19]. In the revised version of the DSM-V [20] (APA), the diagnostic criteria established for minor neurocognitive disorder present a higher positive predictive capacity than the Petersen criteria [21].

So far, the lack of defining functional (in vivo) low-cost biomarkers [22], necessitates the assessment of the disease phenotype and the verification of decline in the patient's cognitive functions [23], through screening tests, for the detection of the disease. It should be noted though that screening tests need interpretation in the context of the full clinical status of the patient and cannot provide a diagnosis on their own. This has 
been emphasized in the diagnostic criteria of Major and Mild Cognitive Impairment in the fifth version of DSM.

\subsection{Screening tests}

Screening tests allow for an assessment of the overall cognitive abilities of older adults. Their administration is brief and can be performed by healthcare personnel after minimal training. Their extended use in populations that are in danger, can reduce healthcare costs [4]. Detection of the possible mild or major neurocognitive disorder by a screening test justifies the cost of neuropsychological assessment and laboratory-clinical tests. Early diagnosis reduces direct (cost of therapy) and indirect (accidents, dangerous behaviour) costs related to the disease. Another issue is the detection of potentially reversible (partially or fully) cognitive decline [24], [25], [26].

To create screening tools that can be applied cross-culturally many tests focusing on assessing key areas of cognition have been created. For instance, using MMSE as an example, evaluation of arithmetic subtraction skill takes place by consecutive arithmetic subtractions, evaluation of time orientation takes place by asking the individual to state the current date etc. This evaluation strategy serves as a template for similar, commonly accepted and valid tests [6], [27]. Adaption and standardization of these tests to fit different target populations minimize the effect of any cultural differences. At the same time, this process preserves the test administration pattern, which remains the same and does not adapt to the cultural characteristics of a population.

Educational attainment can affect the ability of a test to be valid across populations since very low levels of formal education, that can be both a cause and a result of a person's cultural context, can have very significant effects in test performance and even in the feasibility of testing in such a population. At the same time, low educational attainment is not necessarily related to low performance in real-life problem solving (functional intelligence criteria) since low educated older adults often have rich and detailed perception and problem-solving skills, but they are less able to categorize and generalize since these skills are practised in general education.

A part of the scientific community expresses its scepticism regarding the cultural neutrality of tests in general since they have been designed in the context of "western" civilization [28], [29], [30], even though they aim to evaluate the universal "hardcore" (hardware) of human cognition (related to processing speed and accuracy of incoming stimuli, visual and kinetic memory, discrimination, comparison and classification processes). Cognitive pragmatics (software) effects as well as it reflects the content and dynamic effect of the cultural context: language comprehension, reading and skills, formal education, professional skills and knowledge about oneself, and other skills that allow us to distinguish ourselves and cope in daily life [31], [32].

The increasing amount of findings in the domain of cultural neuroscience, where the use of neuroimaging techniques (mainly fMRI) in the evaluation of neuron activity relevant to a person's cultural background, indicates brain areas where this activity has no differences in people among different cultural contexts but also brain areas where neuron activity strongly depends on a person's cultural context [33], [34], [35], [36]. Merging these two important characteristics of human behaviour, "universal" 
(neurophysiological architecture) and "specific" (culturally regulated), appears to be a significant challenge for modern neuropsychology [30] especially in the evaluation of memory and other cognitive functions.

The culturally oriented assessment hypothesis. The sense of stigmatization that often accompanies dementia is often shaped (intensified or mitigated) by culturally established regulative rules that define the attitude of the population concerning ageing, illness, beliefs for the causality of illness, and the socio-cultural experience [4]. One of the major consequences of such stigma is related to the inner motivation of persons to undergo testing of their cognitive functioning. This lack of motivation is added to existing fears of these individuals, concerning the extra burden that will be placed on the family in the hypothetical scenario that they are found to have dementia or even to belong to a high-risk group.

Science and technology are spreading rapidly and homogenously worldwide, providing solutions to crucial problems. They strongly affect how societies and people live and act. This progressive dominance in individual cultural frames [28], rearranges factors like common thinking, emotions, cognition, values, attitudes and beliefs, however, it doesn't minimize their power and effect [37].

Over the last years, there has been a lot of activity in the field of computerized cognitive testing. The advantages of electronic tests are numerous, but it can be argued that the following points provide a concise summary:

a. Very accurate measurement of performance and especially of time metrics such as reaction time.

b. The administration of tests is performed in a standardized format and they provide an unbiased evaluation by negating the effect of different examiners.

c. They require less time for training personnel to administer them (both health experts and auxiliary medical personnel).

d. They can test several cognitive functions in a short amount of time.

e. They are useful for repeat testing offering alternate forms and the ability to easily compare data across administrations.

f. Ability of storing and processing large data sets (which makes them especially useful for large population-wide studies) [38], [39], [40], [41].

\section{Methodology and Material}

Two novel screening tests are used in this study. Both tests are administered through a tablet PC and represent two distinct screening paradigms.

\subsection{Brain health assessment (TabCat)}

The University of California San Francisco (UCSF) Brain Health Assessment (BHA) [42] is a 10-15-minutes tablet-based cognitive assessment battery administered through the Tab CAT software platform. BHA has been designed and developed for iPad devices (optimal screen size around 10") since such devices are very popular in the U.S. 
market. Tab Cat is a secure and friendly platform that is utilized on several international clinical and research sites. It has all the necessary characteristics of a culturally neutral test. The BHA includes tests of memory, executive function, language, and visuospatial skills, and an informant survey, and features automated scoring and guidance for medical follow-up. The areas of cognitive functioning examined by the BHA are related to subtypes of dementia's spectrum disorders. It is designed to provide a quick assessment of cognitive status in primary care and specialized settings. The results of the test regarding the performance of patients in important cognitive functions, together with other clinical findings, constitute reliable indicators of the initiation or the existence of a neurodegenerative disorder.

It can be administered by a non-specialist such as a nurse, social worker or healthcare assistant with minimal training. It follows all the latest trends in computerized test design, it is considered to be an excellent example of the latest generation of computerized tests. It has been validated in a US sample [42] and has shown excellent sensitivity and specificity to MCI and dementia among English speakers (84\% sensitivity for MCI detection vs controls and $100 \%$ sensitivity for dementia detection vs controls when specificity is set at $85 \%$ ). It has been designed as a multilingual test battery and preliminary data support the feasibility of its alternate language versions. BHA further adapted for the Greek population. This version is used in this study.

\subsection{Electronic version of hagia sofia test}

The digital version of Hagia Sophia Test (eHAST) is a culturally oriented screening test aimed at older people whose native language is Greek and have a common cultural background. eHAST has been developed for Android platforms (and is also designed for 10" screens) since the low cost of these devices makes them popular among Greek tablet PC users. The evaluation model differs fundamentally from the majority of computerized and conventional screening tests (excluding components of batteries like RBMT and WAIS which make use of text).

eHAST was developed to bypass dementia-related stigma and increase the proportion of at-risk older adults who are being screened for mild and major neurocognitive disorders in communities who may not necessarily be culturally homogenous but exist within and are aware of the context of this specific myth and the concept of folk myths in general. Culture transcends nationality or any ties to a particular group however living in a certain space with certain predominant cultural characteristics regardless of whether the individual ascribes any meaning or value to them and whether they form parts of the individual's identity. Furthermore, the ultimate goal is to alleviate the inhibitions and fears associated with and accelerated by the cultural incompatibility of the screening strategy that leads to an unwillingness to participate in the screening process, or into false-positive screening results due to poor effort and symptoms of malingering [43].

The test was initially designed to be administered in a pencil and paper format. The pencil and paper version of the test has been administered to a sample of 567 community-based individuals [44]. The conclusions of that study indicate that computerized administration of the test and automated scoring and performance rating will help 
reduce the arbitrary interpretation bias of test answers by various examiners, especially since the test is based on the analysis of verbal responses for the evaluation of the examinee's cognitive function.

At the centre of concern lies the dialectical context of the myth, but not the contained information. Both conventional and electronic administrations dispelled any thoughts on the effect of religious faith. On the other hand, rituals, exist in (or at least are understood as a concept by) every culture. Almost 1500 years have passed since the creation of the Cathedral that is described in the folk myth, and there has been a change on how the temple was originally used in contrast to how it's being used nowadays. Furthermore, its classification into UNESCO's World Heritage Sites has mitigated its connection to a particular religious faith, putting the spotlight on spirituality as a cultural characteristic that is universally known and understood regardless of whether a person adheres to it. The recognizable, common cultural characteristics of a literary text - whether or not the content is known - create a 'familiar' environment for the listener, who is a member of that cultural community. The fact that the myth used in eHAST is one of the oldest but less known myths in Greece, has facilitated that emphasis on the concept and not the content of myth. The text of the myth consists of 128 words and combines realworld knowledge, the negation of that knowledge, and the existence of counterfactuals [45], [46]. The dialectical framework tests lexical-semantic factors, which may affect the process of understanding the semantic links of metaphorical expressions that require a lexically controlled process, as the metaphor has importance in semantic memory. Novel events stimulate attention and trigger increased neuronal activity related to perception, attention, learning, and memory [47]. Computerized administration is fully automated to avoid examiner bias in administration and scoring and to reduce total administration and scoring time. Visual perception [48] is evaluated and the examinee has to fill the missing words in the story which is now dictated sentence by sentence [49].

For the calibration of the test, the semantic and grammatical-syntactic relevance to the target answer was calculated for common answers given by examinees in each question. A perfectly correct word (the word that is originally included in the myth - in its correct grammatical and syntactic form) receives the highest score (30 points). For any other similar or incorrect word(s) the following parameters are taken into account: a) phonetic and phonemic features b) morphological and syntactic features c) semantic features (semantic category, the conceptual relationship of the word with the context, synonyms, hyponyms and meronymy). The maximum score is 540 points. The calibration of the test may be described as exhaustive in an attempt to give as proportionate features as possible.

The design of this culturally oriented screening test for a Greek population that could allow for wider screening among the community was based on several observations:

a. The reluctance of people with low education to be examined with classic pencil and paper tests. They were often unwilling to fully engage in the test process thus their performance often didn't represent their cognitive abilities.

b. Fear of stigma related to being tested for dementia and also fear of receiving a dementia diagnosis 
c. The existence of community centres for older adults (operated by the ministry of health) which can act as hubs for prevention and health promotion programs.

d. The topography. People living in mountainous areas and small islands must expend a lot of time and money to reach a facility, where they can be assessed by a memory specialist. The cost and burden to the older adults and their families should be justified by the results of a standardized test and not just by cognitive concerns. At the same time, this test must be designed to be administered by non-specialists in primary care and community settings.

Since this is a comparative study, the BHA test was chosen in contrast to eHAST as it is a particularly good example of a culturally neutral electronic screening test. It belongs to the latest generation of electronic screening tests for tablets and focuses on early diagnosis of cognitive disorders while at the same time being characterized by brief administration time. Except for the comparison itself, we decided to test eHAST alone (in another sample), to check its performance regarding the clinical diagnoses as well. We ended having data for eHAST vs BHA, and eHAST alone from two independent samples. Each participant was clinically assessed by various instruments and everyone had to accept the agreement form regarding the data collection and personal information.

Inclusion criteria were:

a. 60 years old or more.

b. Greek language as mother tongue.

c. No history of psychiatric disorder or other mental illness that may affect mental functioning.

d. Agreement to sign the informed consent form.

The tests were administered without the examiner is aware of the clinical diagnosis or neuropsychological evaluation results. All participants come from the Greek Association of Alzheimer's Disease and Related Disorders (GAARD) Day Care Centers in Thessaloniki and Alzheimer's Athens. Since different batteries are used to evaluate cognitive functioning in these two sites clinical diagnosis was used to assign subjects to groups.

\section{$3 \quad$ Results}

Our study was based on two approaches. As mentioned, the main goal of this work was to compare two computerized screening tests. But since eHAST is a newly introduced test, we decided to first evaluate its diagnostic utility before moving on to comparisons. For this purpose, we used two different samples correspondingly. We present below the results of these two evaluations. In the first part, we examined the discriminant ability of eHAST based on the clinical diagnosis in a sample of 216 elderly people. In the second part, in a total of 116 subjects, we compared BHA and eHAST in groups of older adults with cognitive concerns OACC-MCI ( $n=56)$ and MCI-Dementia $(n=60)$. We tried to manually balance all groups to achieve as high homogeneity as possible. 


\section{1 eHAST}

As mentioned, eHAST was administered in an independent sample of 216 individuals to evaluate the test's ability to distinguish people with cognitive concerns from those with MCI and dementia. In a total of 216 individuals, the mean age of participants was 74.19 years (S.D. 6.7) and education was 10.69 years (S.D. 4.7). 31.9\% were male (Avg. age 75.92 (S.D. 6.6) and education 12.7 years (S.D. 4.7)) and $68.1 \%$ female (Avg. age 73.3 years (S.D. 6.6) and education 9.7 years (S.D. 4.5)). Thirty-five (35) were in the OACC group having mean age 70.97 years (S.D. 4.87) and 12.91 years education mean ( S.D. 5.2), 114 were in MCI group, with mean age 73.12 years ( S.D. 6.2) and years of education mean 10.39 ( S.D. 4.4), and 67 were in dementia group with mean age 77.7 years ( S.D. 7.01) and years of education mean 10.04 ( S.D. 4.8). Reliability analyses (Cronbach's alpha) was $\alpha=0.905$.

We decided to compare OACC to MCI cases, dementia patients to MCI cases and OACC to Dementia cases as well. For this case, we decided to split MCI cases into 2 groups to match OACC and dementia groups depending on age and education to have as balanced groups as possible. We ended with 30 OACC vs $102 \mathrm{MCI}$ cases, 54 dementia patients vs $90 \mathrm{MCI}$ patients and $25 \mathrm{OACC}$ vs 30 dementia patients.

\subsection{OACC vs MCI}

In the comparison of subjects who were clinically diagnosed in OACC or MCI categories, the mean age of the first group $(n=30)$ was 71.93 years (S.D. 4.3) and the mean education was 12.27 (S.D. 5.37). Respectively for the MCI group $(n=102)$, mean age was 72.62 (S.D. 6.23) and education was 11.10 (S.D. 4.27). Using non-parametric tests ( $\mathrm{p}>.05)$, the distribution of scores, was the same across categories of age and education. Table 1 below shows the results for eHAST's overall score.

Table 1. Descriptive statistics and performance of eHAST test in OACC and MCI

\begin{tabular}{|l|c|c|c|}
\hline & OACC (N = 30) & MCI (N = 102) & P value \\
\hline Gender & M 26.7\% - F 73.3\% & M 27.5\% - F 72,5\% & \\
\hline Age, M (S.D.) & $71.93(4.3)$ & $72.62(6.2)$ & .719 \\
\hline Education, M (S.D.) & $12.27(5.3)$ & $11.10(4.27)$ & .121 \\
\hline eHAST M (S.D.) & $495.76(32.93)$ & $453.33(60.59)$ & .000 \\
\hline Cronbah's $\alpha=0.749$
\end{tabular}

Regarding the ability to distinguish healthy elderly from MCI, eHAST concerning clinical diagnosis was $77,3 \%$ correct (cross-validated).

Table 2. OACC vs MCI eHAST's classification

\begin{tabular}{|l|c|}
\hline & Classification (\% cross validated) \\
\hline eHAST Total & $77,3 \%$ \\
\hline
\end{tabular}




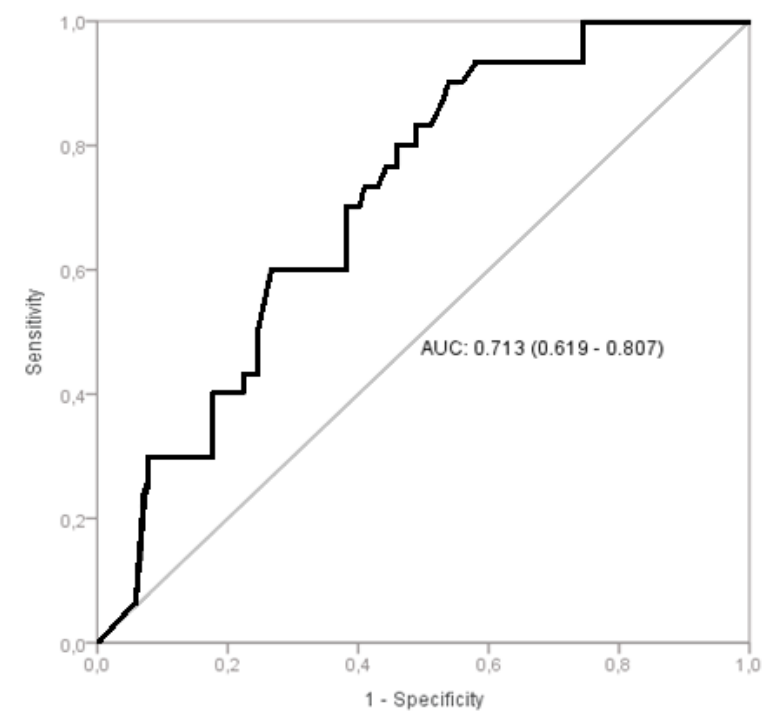

Fig. 1. ROC analyses using eHAST's total score as a predictor

\section{Summary of the ROC analyses}

Area under the curve $=0.713$ (95\% CI: 0.619-0.807)

At best cut-off, sensitivity $=70 \%$, specificity $=63.7 \%$

If sensitivity is set at $80 \%$, specificity $=55.8 \%$

If specificity is set at $56.7 \%$, sensitivity $=79.6 \%$

Dementia vs MCI: In the comparison of subjects with clinical diagnosis classified as MCI and dementia $(n=144)$ the mean age of MCI $(n=90)$ was 74.66 years (S.D. 5.2) while the mean years of education were 10.57 (S.D. 4.37). Respectively for dementia subjects $(\mathrm{n}=54)$, the mean age was 75.64 years (S.D. 5.8) and mean years of education was 10.56 years (S.D. 4.39).

Using non-parametric tests ( $p>.05$ ), the distribution of scores, was the same across categories of age and education. Tables below show the results for eHAST's overall score.

Table 3. Descriptive statistics and performance of eHAST test in MCI and Dementia

\begin{tabular}{|l|c|c|c|}
\hline & MCI (N = 90) & DEMENTIA (N = 54) & P value \\
\hline Gender & M 27.8\% - F 72.2\% & M 42.6\% - F 57.4\% & \\
\hline Age, M (SD) & $74.66(5.2)$ & $75.64(5.8)$ & .083 \\
\hline Education, M (S.D.) & $10.57(4.37)$ & $10.56(4.39)$ & .930 \\
\hline eHAST M (S.D.) & $449.35(61.56)$ & $306.9(108.24)$ & .000 \\
\hline Cronbah's $\alpha=0.894$
\end{tabular}

Regarding the ability to distinguish MCI patients from those with dementia, eHAST concerning clinical diagnosis was $80.6 \%$ correct (cross-validated). 
Table 4. Table MCI vs Dementia eHAST's classification

\begin{tabular}{|l|c|}
\hline & Classification (\% cross validated) \\
\hline eHAST Total & $80.6 \%$ \\
\hline
\end{tabular}

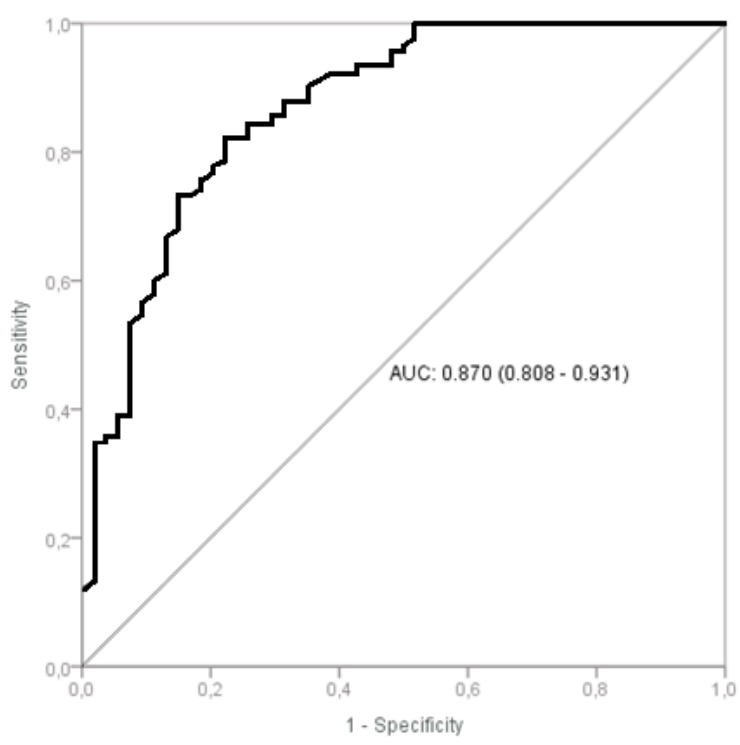

Fig. 2. ROC analyses using eHAST's total score as a predictor

\section{Summary of the ROC analyses}

Area under the curve $=0.870(95 \%$ CI: $0.808-0.931)$

At best cut-off, sensitivity $=82.2 \%$, specificity $=77.8 \%$

If sensitivity is set at $85.6 \%$, specificity $=70.4 \%$

If specificity is set at $85.2 \%$, sensitivity $=73.3 \%$

OACC vs Dementia: In the comparison of subjects with clinical diagnosis classified as OACC and dementia the mean age of OACC $(n=25)$ was 71.0 years (S.D. 4.3) while the mean years of education were 12.24 ( S.D. 4.6), for dementia subjects $(n=30)$, the mean age was 71.86 years (S.D. 4.3) and the mean years of education were 10.90 (S.D. 4.08) Using non-parametric tests ( $\mathrm{p}>.05$ ), the distribution of scores, was the same across categories of age and education, Table 4 below shows the results for eHAST's overall score.

Table 5. Descriptive statistics and performance of eHAST test in OACC and dementia

\begin{tabular}{|l|c|c|c|}
\hline & OACC $(\mathbf{N}=\mathbf{2 5})$ & DEMENTIA $(\mathbf{N}=\mathbf{3 0})$ & P value \\
\hline Gender & M 20\% - F 80\% & M 36.7\% - F 63.3\% & \\
\hline Age, M (SD) & $71.0(4.3)$ & $71.86(4.3)$ & .333 \\
\hline Education, M (SD) & $12.24(4.6)$ & $10.90(4.08)$ & .155 \\
\hline eHAST M (SD) & $492.88(31.92)$ & $308.0(113.01)$ & .000 \\
\hline Cronbah's $\alpha=0.927$
\end{tabular}


Regarding the ability to distinguish MCI patients from those with dementia, e-HAST concerning clinical diagnosis was $89.1 \%$ correct (cross-validated).

Table 6. OACC vs Dementia eHAST's classification

\begin{tabular}{|l|c|}
\hline & Classification (\% cross validated) \\
\hline eHAST Total & $89.1 \%$ \\
\hline
\end{tabular}

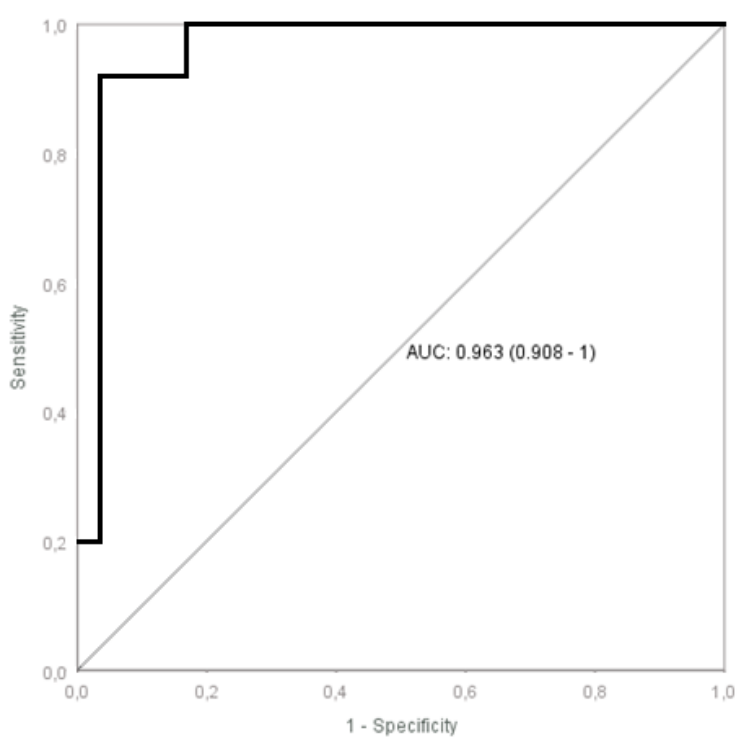

Fig. 3. ROC analyses using eHAST's total score as a predictor

\section{Summary of the ROC analyses}

Area under the curve $=0.963$ (95\% CI:0.908- 1)

At best cut-off, sensitivity $=92 \%$, specificity $=96.7 \%$

If sensitivity is set at $100 \%$, specificity $=83.3 \%$

If specificity is set at $100 \%$, sensitivity $=80 \%$

\subsection{BHA vS eHAST}

OACC vs MCI: In the comparison between OACC vs MCI groups, 56 individuals participated with mean ages 71.29 years (S.D. 4.6) and education 11.7 years (S.D. 3.9), $23.2 \%$ of them were male with mean age 71.62 years (S.D. 5.0) and mean years of education was 12.54 (S.D. 4.5) and $76.8 \%$ were female with mean age 71.19 years (S.D. 4.5) and education 11.5 years (S.D. 3.8).

The mean age of the subjects with clinical diagnosis classified as OACC $(n=19)$ was 71.37 years (S.D. 4.0) while the mean of years of education was 12.16 (S.D. 3.4). Respectively for MCI $(n=37)$, the mean age was 71.24 years (S.D. 4.9) and mean years of education was 11.57 (S.D. 4.2). 
Using non-parametric tests ( $\mathrm{p}>.05)$, the distribution of scores, both for BHA and eHAST, was the same across categories of age and education. Table 4 below shows the results for each one of BHA's subtests and eHAST's overall score.

Table 7. Descriptive statistics and performance of BHA and HAST tests in OACC and MCI

\begin{tabular}{|l|c|c|c|}
\hline \multicolumn{1}{l|}{} & OACC $(\mathbf{N}=\mathbf{1 9})$ & MCI $(\mathbf{N}=\mathbf{3 7})$ & P value \\
\hline Gender & M 21.1\% - F 78.9\% & M 24.3\% - F 75.7\% & \\
\hline Age, M (SD) & $71.37(4.03)$ & $71.24(4.95)$ & .920 \\
\hline Education, M (SD) & $12.16(3.45)$ & $11.57(4.27)$ & .580 \\
\hline Favorites Total, M (SD) & $11.53(5.37)$ & $9.51(6.74)$ & .231 \\
\hline Match Total, M (SD) & $40.21(7.53)$ & $33.39(10.52)$ & .008 \\
\hline Animal Fluency, M (SD) & $20.63(4.88)$ & $15.05(5.72)$ & $<.001$ \\
\hline Line Length, M* (SD) & $3.76(1.84)$ & $3.64(1.94)$ & .822 \\
\hline eHAST M (SD) & $485.21(40.08)$ & $452.94(64.46)$ & .026 \\
\hline
\end{tabular}

*Greater values indicated worse performance

Regarding the ability to distinguish OACC from MCI, BHA's overall sub-tests concerning clinical diagnosis was $64.7 \%$ (cross-validated) while eHAST concerning clinical diagnosis was $60,7 \%$ correct (cross-validated).

Table 8. BHA subtests vs eHAST Discriminant Analyses

\begin{tabular}{|l|c|}
\hline & Classification (\% cross validated) \\
\hline Favourites Total, M (SD) & 60.7 \\
\hline Match Total, M (SD) & 67.3 \\
\hline Animal Fluency, M (SD) & 67.9 \\
\hline Line Length, M (SD) & 63 \\
\hline BHA Total & 64.7 \\
\hline eHAST & 60.7 \\
\hline
\end{tabular}

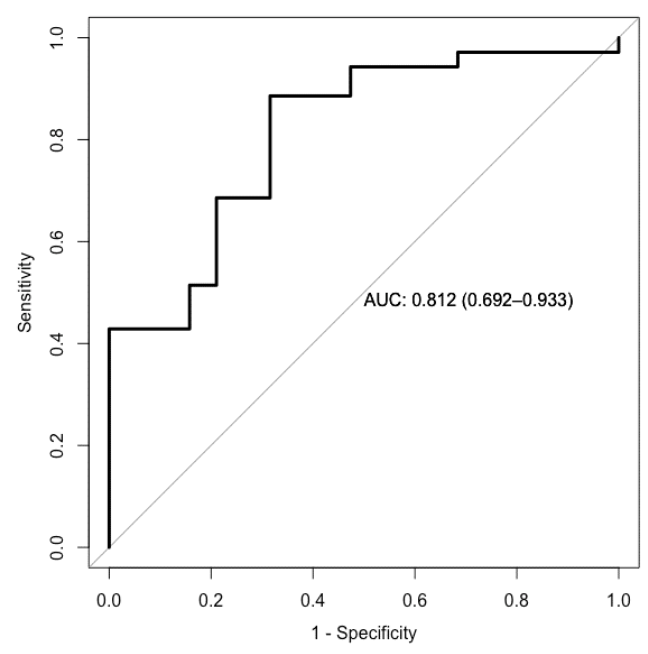

Fig. 4. Control vs MCI ROC analyses using BHA's raw scores of all four tests as predictors 


\section{Summary of the ROC analyses}

Area under the curve $=0.812(95 \%$ CI:0.692-0.933)

At best cut-off, sensitivity $=89 \%$, specificity $=68 \%$

If sensitivity is set at $85 \%$, specificity $=68 \%$

If specificity is set at $85 \%$, sensitivity $=43 \%$

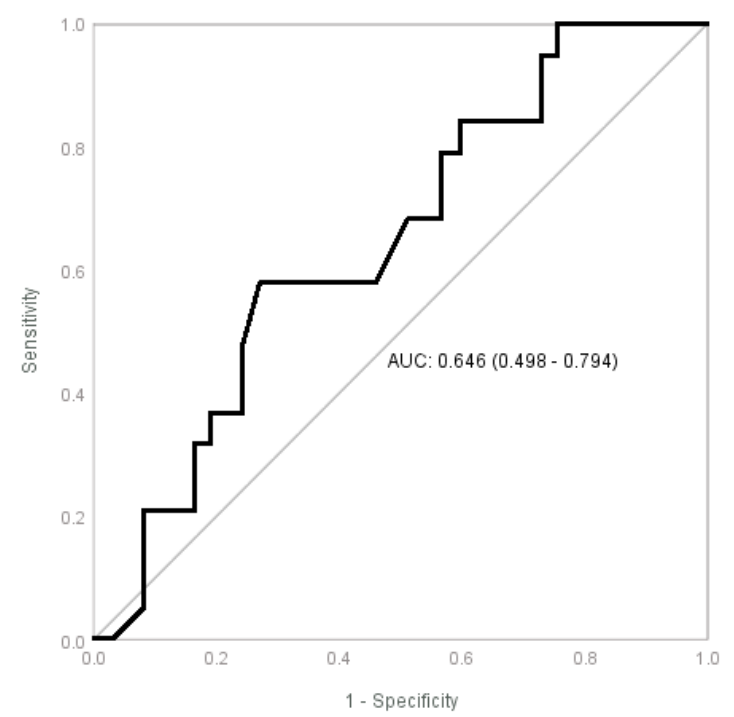

Fig. 5. Control vs MCI ROC analyses using eHAST's total score as a predictor

\section{Summary of the ROC analyses}

Area under the curve $=0.646$ (95\% CI:0.498- 0.794)

At best cut-off, sensitivity $=57,9 \%$, specificity $=73 \%$

If sensitivity is set at $78,9 \%$, specificity $=40,5 \%$

If specificity is set at $75,7 \%$, sensitivity $=47,4 \%$

MCI vs Dementia: In MCI and Dementia groups participated 60 individuals with mean age 74.77 years (S.D. 5.8) and mean years of education 10.2 (S.D. 4.3), 31.7\% of them were male with mean age 75.95 years (S.D. 6.6) and mean years of education 13.32 (S.D. 4.4) and 68,3\% were female with mean age 74.22 years (S.D. 5.3) and mean years of education 8.8 years (S.D. 3.6).

The mean age of the subjects with clinical diagnosis classified as MCI $(n=39)$ was 74.72 years (S.D. 6.55) while the mean years of education were 10.18 (S.D. 4.42). Respectively for dementia patients $(n=21)$, the mean age was 74.86 years (S.D. 4.23) and mean years of education 10.33 (S.D. 4.43).

Using non-parametric tests ( $p>.05)$, the distribution of scores, both for BHA and eHAST, was the same across categories of age and education. Table 6 below shows the results for BHA's subtests and e-HAST's overall score. 
Paper-Tablet-Administered Screening Tests for the Detection of Major and Mild Cognitive..

Table 9. Descriptive statistics and performance of BHA and HAST tests in MCI and Dementia

\begin{tabular}{|l|c|c|c|}
\hline & MCI (N = 39) & DEMENTIA (N = 37) & P value \\
\hline Gender & M 28.2\% - F 71.8\% & M 38.1\% - F 61.9\% & \\
\hline Age, M (S.D.) & $74.72(6.55)$ & $74.86(4.23)$ & .921 \\
\hline Education, M (S.D.) & $10.18(4.42)$ & $10.33(4.43)$ & .898 \\
\hline Favorites Total, M (S.D.) & $8.69(6.26)$ & $5.43(4.64)$ & .026 \\
\hline Match Total, M (S.D.) & $30.58(9.96)$ & $20.47(16.49)$ & .021 \\
\hline Animal Fluency, M (S.D.) & $13.92(5.71)$ & $12.38(6.79)$ & .382 \\
\hline Line Length, M* (S.D.) & $3.77(1.9)$ & $4.14(2.76)$ & .607 \\
\hline eHAST M (S.D.) & $434.48(64.36)$ & $350.57(95.19)$ & .001 \\
\hline
\end{tabular}

*Greater values indicated worse performance

Regarding the ability to distinguish MCI patients from those with dementia, BHA's overall sub-tests concerning clinical diagnosis was $66.1 \%$ correct (cross-validated), while eHAST concerning clinical diagnosis was $76.7 \%$ correct (cross-validated).

Table 10. BHA subtests vs eHAST Discriminant Analyses

\begin{tabular}{|l|c|}
\hline & Classification (\% cross validated) \\
\hline Favourites Total, M (S.D.) & 63.3 \\
\hline Match Total, M (S.D.) & 77.2 \\
\hline Animal Fluency, M (S.D.) & 65 \\
\hline Line Length, M (S.D.) & 66.7 \\
\hline BHA Total & 66.1 \\
\hline eHAST & 76.7 \\
\hline
\end{tabular}

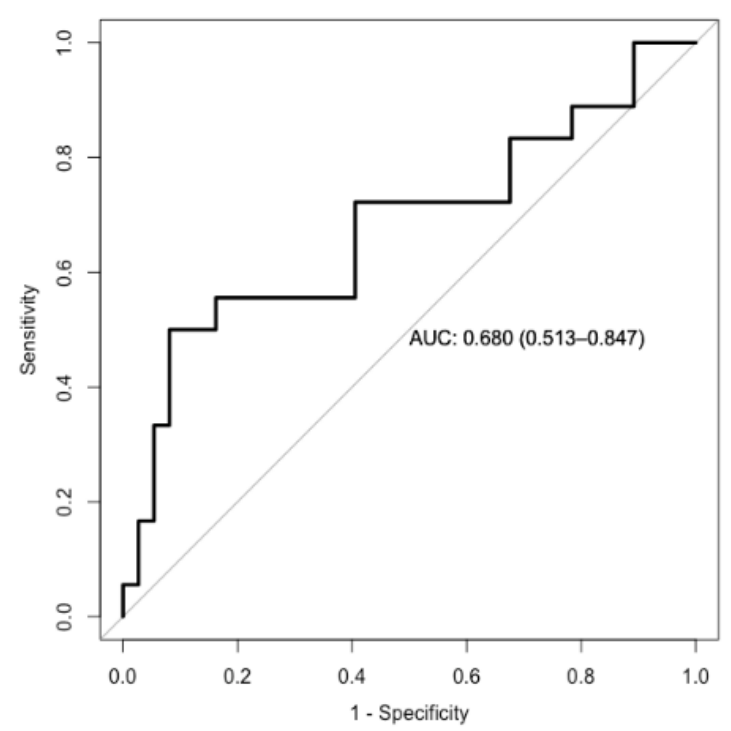

Fig. 6. MCI vs Dementia ROC analyses using BHA's raw scores of all four tests as predictors 


\section{Summary of the ROC analyses}

Area under the curve $=0.680$ (95\% CI: $0.513-0.847)$

At best cut-off, sensitivity $=50 \%$, specificity $=92 \%$

If sensitivity is set at $85 \%$, specificity $=22 \%$

If specificity is set at $85 \%$, sensitivity $=50 \%$

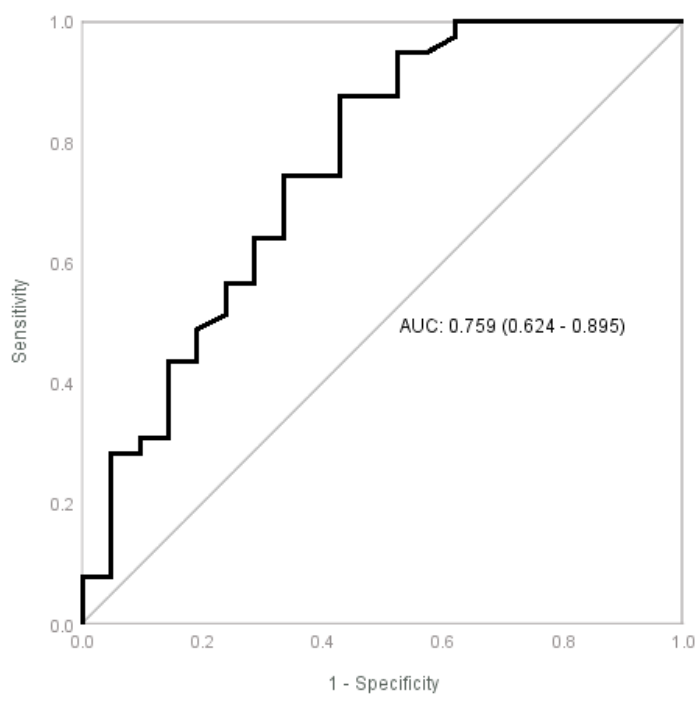

Fig. 7. MCI vs Dementia ROC analyses using eHAST's total score as a predictor

\section{Summary of the ROC analyses}

Area under the curve $=0.759(95 \%$ CI: $0.624-0.895)$

At best cut-off, sensitivity $=74,4 \%$, specificity $=66,7 \%$

If sensitivity is set at $87,2 \%$, specificity $=57,1 \%$

If specificity is set at $76,2 \%$, sensitivity $=56,4 \%$

\section{Discussion}

In this study, the performance of two computerized tests was evaluated concerning their ability to differentiate between a) older adults with cognitive concerns (OACC) and those with MCI, b) adults with MCI and those with dementia. The structure and strategy of examination of the two tests differ significantly.

The examination of the diagnostic accuracy of these two tests in comparison to clinical diagnosis is not intended to promote them as alternatives to a diagnosis by a specialist. Screening acts only as a starting point for referral to specialist assessment. A positive screening result, however, justifies the need (and subsequent cost) of specialized assessment [6].

Regarding eHAST, the results from a sample of 216 participants indicate a really good discriminant ability between groups of OACC and dementia. eHAST was 
originally designed as a screening test particularly for major cognitive disorders, and it seems that it fulfils this purpose greatly. The exploratory analysis shows high discriminant ability between groups of MCI and dementia as well but has low discriminant ability between OACC and MCI groups.

At the same time, it should be noted that differentiating between OACC and MCI patients is difficult, even for trained clinicians, as differences in cognitive performance can be subtle and the underlying biological background may differ greatly among individuals. The same study should be replicated in a larger sample, to get more valid results.

It should be noted that while eHAST global score was used in this study we chose to use BHA domain scores (on their own and in combination when used in a classifier model) since this test has not been normed in a Greek population and therefore a global score cannot be calculated accurately. Furthermore, the BHA Match subtest performed consistently well in the differentiation between OACC and MCI patients and between MCI patients and dementia patients. The match is a measure of executive function thus these results indicate the need for the comprehensive evaluation of multiple domains, even in screening batteries, as memory is often not the best indicator of cognitive status. BHA has almost $7 \%$ higher discriminant ability compared to eHAST, when differentiating between OACC- MCI groups, while they present almost similar discriminant ability when differentiating between MCI-dementia groups. Based on a classifier model when the eHAST global score and the BHA match score are the group for discrimination between groups respectively. Our findings for BHA differ from those by Possin et al [42] study where the test exhibits $84 \%$ sensitivity for MCI detection and $100 \%$ sensitivity for dementia both in comparison with healthy older adults in US population. We can attribute this to the characteristic of our sample which comprises less-educated older adults with cognitive concerns and who are unfamiliar with technology versus the US study's sample which comprises highly educated older adults with no cognitive concerns and high levels of technological literacy. Furthermore, a large normative sample exists for the US population thus allowing for detailed norming of the BHA further enhancing its ability to detect abnormal cognition. Additionally, we were not able to calculate the effect of the BHA informant survey (Brain Health Survey - BHS) in its diagnostic accuracy as participants did not enter the study along with a relative that could act as an informant. Results from the US BHA study indicate that the BHS increases the BHA's diagnostic accuracy [42]. Sensitivity and specificity of eHAST are lower than BHA but requires less administration time (10') approaching the 5-7 minutes limit [50], [51], while BHA requires 5' more, in a total of 15 minutes.

In Greece, there has been an effort to validate a serious game-based cognitive screening application, based on a virtual shopping task, the Virtual Supermarket (VSM) [52]. It has displayed a high discriminant ability $(87.3 \%)$ when differentiating between groups of healthy older adults with cognitive concerns and MCI patients. It represents another approach to deviating from the standard cognitive screening paradigm that is based on a traditional cognitive test. The use of an alternative scenario like a daily activity instead of a conventional neuropsychological testing model increases its ecological validity in the hope of negating cultural barriers to screening and mitigating the effect of unfamiliarity with standardized testing procedures. 
Regarding metric ability in comparison to other tablet-based tests in iOS or Android:

a. eHAST presents a lower performance in sensitivity and specificity between health MCI groups - compared to Computer Assessment of Memory and Cognitive Impairment (CAMCI) [53], Mobile Screening System for Mild Cognitive Impairments (mSTS-MCI) [54], Cambridge Neuropsychological Test Automated Battery (CANTAB MOBILE) [55], Visual Super Market (VSM) [52].

b. BHA performance in the present study is lower than CAMCI, mSTS-MCI, VSM, while being superior in sensitivity but lacking in sensitivity and specificity in comparison to CANTAB MOBILE.

c. eHAST performs poorly compared to Cognitive Assessment for Dementia (CADi2) [56] and Computerized Cognitive Screening (CCS) [57] between MCI and Demented. BHA (in the present study) significantly exceeds sensitivity to CCS.

None of these tests, however, has been tested in a Greek population and their discriminant ability is calculated on healthy older adults (Vs MCI/ dementia) when the typical population of older adults that seek screening have subjective memory complaints (even in the absence of objective impairment). By trialling two novel tests, each representing a different screening paradigm, this study aims to contribute to the validation and subsequent implementation of screening tools that are relevant to and usable with Greek older adults.

Regarding future directions, as mentioned, initially eHAST was designed to distinguish between healthy older adults and elderly with dementia. The intrinsic characteristics of the myth's text and the ability to exploit the enormous amount of data given the computational power of the device offered the prospect of testing its ability to detect MCI. The results of the comparison between eHAST and BHA and the results of studies on other tests administered by mobile devices can be the starting point for future studies whose overall aim would be to pave the way for widespread implementation of computerized screening in Greece. A first step could be increasing the sample to evaluate the metric power of the two tests in the Greek population as the difference in average age between OACC and dementia subjects in the present study did not allow us to accurately calculate its discriminant ability for differentiating between these groups. At the same time, the use of fluent speech during its representation could be used as a novel marker to identify other types of dementia as speech disorders are found in Alzheimer's disease, vascular dementia, frontotemporal dementia and Lewy body dementia. Furthermore, the inclusion of a healthy community-dwelling older adult sample with no cognitive concerns could allow for robust norming of both tests in the Greek population. Lastly, implementation studies in "real-world" community and clinical settings would allow for an assessment of the benefits of these tests for the healthcare system.

Finally, we should note the limitations of the study, as we acknowledge that the sample is small to conclusively evaluate the ability of both tests in differentiating between groups. Normative studies in a larger sample of diverse age and education will provide the ability to establish normative data and assess in detail the effect of age and education. 


\section{Conclusion}

We are facing an important challenge. The prevalence of dementia in developing countries will be increased significantly in the coming years, especially in Africa and Asia (76.7\% of the world population [58]). Poverty is accompanied by low levels of education and in some regions, it is combined with rigid regulatory social norms. The stigma is higher in these societies where a neurodegenerative brain disease can result in shame, shunning, social exclusion and even accusations of witchcraft. There is an abundance of accurate cognitive screening tests for the detection of mild or major neurocognitive disorders however, globally, dementia remains underdiagnosed [4], [5], [6].

Experimental use of a culturally oriented screening test for a high-income country's population which combines cultural characteristics of 'western' society with its unique culturally specific features in, a well-forged through aeons, the specific cultural context seems incompatible to these aforementioned populations. At the same time the importance of the screening paradigm being introduced, and the power of folk myths to be universally understandable and relatable must be highlighted. In G20 countries with huge populations such as China and India (36.3\% of the world population) and with correspondingly long-lived cultures where a large proportion of the population is poorly educated and therefore has low living standards, we expect an increase in life expectancy and consequently increase in cases of dementia. In both countries, the value of evaluating cognitive functions in the elderly has been recognized. As Woo J. [11] points out, 'Much work needs to be done to select a tool for a specific ethnic group distinguishing educational level (for different levels of education) as well as integrating evaluation into health systems'. The hypothesis of alternative ecologically valid and culturally oriented screening tests could be a pathway to an effective response to this, ever-intensifying, health problem concerning cultural context while at the same time adhering to established scientific standards.

The use of a tablet pc in the assessment of cognitive disorders in the elderly can have a catalytic role in the struggle against dementia. The portability allows their use both inside and outside the clinic and in epidemiological studies. Factors such as location, time, and frequency of administration are mitigated. Some applications allow the involvement of health professionals not specialized in psychometry or/ and people of the elderly environment. This allows us to increase the percentage of older people screened and monitoring.

Physical limitations such as vision deficiencies and upper limb mobility, blunted using screen 9.7-10.1 " while maintaining portability. The visual and auditory formulation of the instructions minimizes the differences in administration. The use of eye-tracker for the design of interface and multimedia contributes decisively. Data recording (reaction time, stylus pen trace, audio recording etc.) and the exhaustive calibration of the tests increase the qualitative and quantitative data which are available to the clinician. The increasing computing power of mobile devices may allow us the use of deep learning by integrating Artificial Intelligence networks to adapt the psychometric tasks not only to the extent of the cognitive functions of the patient but furthermore to the assessment based on the subtype of dementia disorder. 
The preliminary comparison of eHAST, a culturally oriented screening test, with the state of the art culturally-neutral BHA test, aims to become a starting point for the examination of alternative, technology-based screening paradigms that blend the "hard" science of neuropsychology with the cultural sensitivity needed for approaching older adults in their context. The verification of the initial hypothesis can further lead to the creation of an algorithm of characteristics that must be shared by the various myths of the cultural groups we wish to examine through myth-based, culturally oriented cognitive tests. The overarching goal is to mitigate prohibitions that arise due to fear of stigmatization, low level of education, or the clash with norms, and in general with the way of thinking and acting at a specific cultural environment.

\section{Acknowledgement}

The development of the electronic application and the described comparative study are parts of an overall project which is co-funded by Greek and the European Union (European Social Fund) through the Operational Program "Human Resources Development, Education and Lifelong Learning 2014-2020". MIS 5005195. The authors would like to thank Dr Katherine Possin, Dr Elena Tsoy, PhD Stelios Zygouris and all the BHA team for their invaluable assistance and support. The authors report no conflict of interest.

\section{$7 \quad$ References}

[1] Alzheimer's Disease International. (2018). The state of the art of dementia research: New frontiers. Available: https://www.alz.co.uk/research/world-report-2018

[2] Alzheimer's Disease International (2015). The Global impact of dementia. An analysis of prevalence, incidence, cost and trends. World Alzheimer Report 2015.Available: http:// www.alz.co.uk/research/WorldAlzheimerReport2015.pdf

[3] World Health Organization. The epidemiology and impact of dementia. Current state and future trends. Available: http://www.who.int/mental_health/neurology/dementia/thematicbrief_epidemiology.pdf

[4] World Alzheimer Report 2019: Attitudes to dementia Available: https://www.alz.co.uk/research/world-report-2019

[5] H. Frankish and R. Horton, 'Prevention and management of dementia: a priority for public health.' Lancet (London, England), Vol. 390(10113) pp2614-2615, Dec. 2017.Available: https://doi.org/10.1016/s0140-6736(17)31756-7

[6] B. Cullen, B. O'Neill, J.J. Evans, R.F. Coen, B.A. Lawlor, 'A review of screening tests for cognitive impairment.' Journal of Neurology, Neurosurgery \& Psychiatry, Vol.78, pp790799, 2007. https://doi.org/10.1136/jnnp.2006.095414

[7] H. Brodaty, J.Clarke, M. Ganguli, et al. 'Screening for cognitive impairment in general practice: toward a consensus.' Alzheimer Disease and Associated Disorders, Vol. 12(1), pp1-13, Mar 1998. https://doi.org/10.1097/00002093-199803000-00001

[8] J.S. Lin, E. O’Connor, R.C. Rossom, L.A., Perdue, B.U. Burda, M. Thompson, E. Eckstrom 'Screening for Cognitive Impairment in Older Adults: A Systematic Review for the U.S. Preventive Services Task Force. Rockville (MD): Agency for Healthcare Research and 
Quality (US).' Ann Intern Med, Vol.159, pp 601-612, 2013.Available: https://doi. org/10.7326/0003-4819-159-9-201311050-00730

[9] M. Tsolaki, P. Sakka, N. Degleris, S. Karamavrou. 'Attitudes and perceptions regarding Alzheimer's disease in Greece.' Am J Alzheimers Dis Other Demen, Vol 24(1), pp21-6, 2009. https://doi.org/10.1177/1533317508325990

[10] M. Boustani, B. Peterson, L. Hanson, R. Harris, K.N. Lohr, 'U.S. 'Preventive Services Task Force. Screening for dementia in primary care: a summary of the evidence for the U.S. Preventive Services Task Force.' Ann Intern Med, Vol. 138(11), pp927-37, 2003. https://doi.org/10.1037/e554792010-001

[11] J. Woo, 'Brain Health: The Importance of Recognizing Cognitive Impairment: An Asian Viewpoint.' Journal of the American Medical Directors Association, Vol. 16, Issue 9, pp 722-723, 2015 Available: https://doi.org/10.1016/j.jamda.2015.06.003

[12] D.M. Rentz, M. Dekhtyar, J. Sherman, S. Burnham, D. Blacker, S.L. Aghjayan, K.V. Papp, R.E. Amariglio, A. Schembri, T. Chenhall, P. Maruff, P. Aisen, B.T. Hyman, R.A. Sperling 'The Feasibility of At-Home iPad Cognitive Testing For Use in Clinical Trials.' The journal of prevention of Alzheimer's disease, Vol3(1), pp8-12, Mar. 2016.

[13] H.I. Makizako, H. Shimada, H. Park, T. Doi, D. Yoshida, K. Uemura, K.Tsutsumimoto, T. Suzuki, 'Evaluation of multidimensional neurocognitive function using a tablet personal computer: test-retest reliability and validity in community-dwelling older adults.' Geriatr Gerontol Int, Vol.13(4), pp860-866, Okt 2013. https://doi.org/10.1111/ggi.12014

[14] E.C. Edmonds L.Delano-Wood, L.R. Clark, A.J. Jak, D.A. Nation, C.R. McDonald et al, 'Susceptibility of the conventional criteria for mild cognitive impairment to false-positive diagnostic errors.' Alzheimer's \& Dementia, Vol11(4), pp. 415-24, Apr. 2015. https://doi. org/10.1016/j.jalz.2014.03.005

[15] R.C. Petersen, R. Doody, A. Kurz, et al., 'Current concepts in mild cognitive impairment: clinical characterization and outcomes.' Arch Neurol, Vol.58, p.p.1985-1992, 2001

[16] B. Winblad, K. Palmer, M. Kivipelto et al, 'Mild cognitive impairment beyond controversies, towards a consensus: report of the International Working Group on Mild Cognitive Impairment.' J Intern Med, Vol.256(3), pp 240-246, Sept 2006. https://doi.org/10.1111/j. 1365-2796.2004.01380.x

[17] M. Bruscoli and S. Lovestone, 'Is MCI really just early dementia? A systematic review of conversion studies.' International Psychogeriatrics, Vol.16(2), pp 129-140, June 2004 https://doi.org/10.1017/s1041610204000092

[18] A.J. Mitchell and M. Shiri-Feshki 'Rate of progression of mild cognitive impairment to dementia meta-analysis of 41 robust inception cohort studies.' Acta Psychiatr Scand, Vol.119, pp 252-265, Apr. 2009. https://doi.org/10.1111/j.1600-0447.2008.01326.x

[19] S. Gauthier, B. Reisberg, M. Zaudig et al, 'Mild cognitive impairment.' The Lancet, Vo.367 (9518), pp 1262-1270, April 2006.

[20] American Psychiatric Association (2013). 'Diagnostic Criteria from DSM-5'. American Psychiatric Publishing Washington, DC London England p. 299-301

[21] N.I. Bradfield and D. Ames, 'Mild cognitive impairment: narrative review of taxonomies and systematic review of their prediction of incident Alzheimer's disease dementia.' B.J.Psych Bul, Vol.14, pp1-8, 2019. https://doi.org/10.1192/bjb.2019.77

[22] Biomarkers Definitions Working Group 'Biomarkers and surrogate endpoints: preferred definitions and conceptual framework.' Clinical Pharmacology and Therapeutics Vol.69 (3) pp 89-95. March 2001. https://doi.org/10.1067/mcp.2001.113989

[23] K. Ritchie and D. Kildea 'Is senile dementia "age-related" or "ageing-related"? evidence from meta-analysis of dementia prevalence in the oldest old.' Lancet, Vol.346(8980), pp 931-934, Oct. 1995. https://doi.org/10.1016/s0140-6736(95)91556-7 
[24] J.E. Morley, J.C. Morris, M. Berg-Weger et al, 'Brain health: the importance of recognizing cognitive impairment: an IAGG consensus conference.' J Am Med Dir Assoc, Vol.16(9), pp 731-739, Sept 2015.

[25] M.D. Weytingh, P.M.M. Bossuyt, H. van Crevel, 'Reversible dementia: more than $10 \%$ or less than 1\%?' J Neurol, Vol. 242, pp 466 - 471, 1995. https://doi.org/10.1007/bf00873 $\underline{551}$

[26] O. Emery, 'Pseudodementia: A Theoretical and Empirical Discussion: Interdisciplinary Monograph Series.' In G.S. Alexopoulos, B.S.Meyers, R.C. Young, S. Mattis, T. Kakuma, 'The course of geriatric depression with "reversible dementia": a controlled study.' Am J Psychiatry, Vol. 150(11), pp1693-1699, Nov. 1993 https://doi.org/10.1176/ajp.150.11. $\underline{1693}$

[27] K.K. Tsoi, J.Y. Chan, H.W. Hirai, S.Y. Wong, T.C. Kwok, 'Cognitive Tests to Detect Dementia: A Systematic Review and Meta-analysis.' JAMA Intern Med, Vol.175(9),p.p.14501458, Sept 2015. https://doi.org/10.1001/jamainternmed.2015.2152

[28] A. Ardila, 'Cultural Values Underlying Psychometric Cognitive Testing.' Neuropsychology review, Vol.15, Pp 185-195, Dec. 2005. https://doi.org/10.1007/s11065-005-9180-y

[29] C.G. Matthews, 'Truth in labeling: are we really an international society?' J Clin Exp Neuropsychol, Vol. 14(3), pp418-426, Jun. 1992.

[30] N.W. Nelson and M.O. Pontón, The Art of Clinical Neuropsychology from: International Handbook of Cross-Cultural Neuropsychology.' Routledge Chapter 3 pp 5-62. Jan 2007, Accessed on 09 Mar 2020 https://www.routledgehandbooks.com/doi/10.4324/978020 3936290.ch0003

[31] D.C. Geary and J. Lin, 'Numerical cognition: age-related differences in the speed of executing biologically primary and biologically secondary processes.' Exp Aging Res, Vol.24(2), pp101-137. Apr-Jun 1998 https://doi.org/10.1080/036107398244274

[32] P.B. Baltes, 'The Aging Mind: Potential and Limits.' The Gerontologist, Vol. 33 (5), pp 580-594, Oct 1993. https://doi.org/10.1093/geront/33.5.580

[33] S. Han and G. Northoff. 'culture-sensitive neural substrates of human cognition: a transcultural neuroimaging approach.' Nat Rev Neurosci, Vol.9(8), pp.646-54, Aug.2008 https://doi.org/10.1038/nrn2456

[34] D.C. Park and C.M. Huang, 'Culture Wires the Brain: A Cognitive Neuroscience Perspective.’ Perspect Psychol Sci, Vol. 5(4), pp 391-400, Jul 2010.

[35] S. Han and Y. Ma, 'Cultural differences in human brain activity: a quantitative meta-analysis.’ Neuroimage, Vol.99, pp293-300, Oct 2014. https://doi.org/10.1016/j.neuroimage. $\underline{2014.05 .062}$

[36] Y. Zhu, L. Zhang, J. Fan, S. Han, 'Neural basis of cultural influence on self-representation.' Neuroimage, Vol.34(3), pp 1310-1316, Feb. 2007. https://doi.org/10.1016/j.neuroimage.2006.08.047

[37] J. Adamopoulos, 'Interpersonal behaviour: Cross-cultural and historical perspectives.' In: 'The Cross-Cultural Challenge to Social Psychology.' M.H. Bond, Ed, Sage publications, 1988, pp. 196-207.

[38] S. Zygouris and M. Tsolaki, 'Computerized cognitive testing for older adults: a review'. Am J Alzheimers Dis Other Demen. Vol.30(1), pp13-28, Feb. 2015. https://doi.org/10. $\underline{1177 / 1533317514522852}$

[39] B.M. Koo and L.M. Vizer, 'Mobile Technology for Cognitive Assessment of Older Adults: A Scoping Review.' Innovation in Aging, Vol.3(1), igy038, Jan 2019. https:// doi.org/10.1093/geroni/igy038

[40] S.B. Thacker and R.L. Berkelman 'Public health surveillance in the United States'. Epidemiol Rev, Vol.10, pp.164-190, 1988 
[41] A. Milne, A. Culverwell, R. Guss, J. Tuppen, R. Whelton 'Screening for dementia in primary care: a review of the use, efficacy and quality of measures.' Int. psychogeriatrics, Vol. 20 (5), pp. 911-926, Oct 2008. https://doi.org/10.1017/s1041610208007394

[42] K.L. Possin, T. Moskowitz, S.J. Erlhoff, K.M. Rogers, E.T. Johnson, N.Z.R. Steele, J.J. Higgins, J. Stiver, A.G. Alioto, S.T. Farias, B.L. Miller, K.P. Rankin, 'The Brain Health Assessment for Detecting and Diagnosing Neurocognitive Disorders.' J Am Geriatr Soc. Vol. 66(1), pp.150-156, Jan. 2018. https://doi.org/10.1111/jgs.15208

[43] G.L. Iverson, 'Ethical Issues Associated With the Assessment of Exaggeration, Poor Effort, and Malingering.' Journal Applied Neuropsychology, Vol. 13 (2), pp 77-90, 2006. https://doi.org/10.1207/s15324826an1302 3

[44] A.A. Solias, N.E. Degleris, I. Kompatsiaris, A. Tsangalidis, L.J. Hadjileontiadis, M. N. Tsolaki, 'A folk legend for the detection of dementia: an active part of online medical data entry channel.' Hell J Nucl Med Suppl, Vol.20(2) pp 169-179, May Aug. 2017 Available at: https://www.nuclmed.gr/wp/wp-content/uploads/2017/07/Supplement.pdf. $\quad$ https://doi. org/10.18411/a-2017-023

[45] H.J. Ferguson, A.J. Sanford, H. Leuthold, 'Eye-movements and ERPs reveal the time course of processing negation and remitting counterfactual worlds.' Brain Res. Vol. 1236 pp.11325, Oct 2008. https://doi.org/10.1016/j.brainres.2008.07.099

[46] C. Lévi-Strauss, "The Structural Study of Myth." The Journal of American Folklore, vol. 68, no. 270, pp. 428-444,1955. https://doi.org/10.2307/536768

[47] V. Bonnaud, R. Gil, P. Ingrand P., 'Metaphorical and non-metaphorical links: a behavioural and ERP study in young and elderly adults.' Neurophysiol Clin, Vol.32(4), pp.258-68, Sept 2002. https://doi.org/10.1016/s0987-7053(02)00307-6

[48] L. Ghent 'Perception of overlapping and embedded figures by children of different ages.' New York University 1955, p575-587.

[49] A.A. Solias, A.E. Chaldogeridis, A.V., Batzikosta, M.N.Tsolaki, 'Comparison of two electronic screening tests (culturally neutral \& culturally customized) for major and minor neurocognitive disorders.' Hell J Nucl Med., 22 Suppl, pp111-122, Jan. 2019.

[50] J.E. Morley, J.C. Morris, M. Berg-Weger, S. Borson, et al, 'Brain health: the importance of recognizing cognitive impairment: an IAGG consensus conference.' J Am Med Dir Assoc, Vol.16(9), pp. 731-739, Sept. 2015.

[51] R.W. Aslam, V. Bates, Y. Dundar, J. Hounsome, Richardson M, Krishan A, Dickson R, Boland A, Fisher J, Robinson L, Sikdar S, 'A systematic review of the diagnostic accuracy of automated tests for cognitive impairment.' Int J Geriatr Psychiatry, Vol. 33(4), pp. 561575, April 2018. https://doi.org/10.1002/gps.4852

[52] S. Zygouris, D. Giakoumis, K. Votis, St. Doumpoulakis, K. Ntovas, S. Segkouli, Ch. Karagiannidis, D. Tzovaras, M. Tsolaki, 'Can. A Virtual Reality Cognitive Training Application Fulfill a Dual Role? Using the Virtual Supermarket Cognitive Training Application as a Screening Tool for Mild Cognitive Impairment', Journal of Alzheimer's Disease, vol. 44 (4), pp. 1333-1347, 2015. https://doi.org/10.3233/jad-141260

[53] J. Saxton, L. Morrow, A. Eschman, G. Archer, J. Luther, A. Zuccolotto, 'Computer Assessment of Mild Cognitive Impairment.' Postgraduate Medicine, Vol. 121(2), pp. 177- 185, 2009. https://doi.org/10.3810/pgm.2009.03.1990

[54] J.H. Park, M. Jung, J. Kim, H.Y. Park, 'Validity of a novel computerized screening test system for mild cognitive impairment.' International Psychogeriatrics, Vol.30(10), pp. 1455-1463, Oct 2018. https://doi.org/10.1017/s1041610218000923

[55] J. Chandler, M. Marsico, L. Harper-Mozley, R. Vogt, Y. Peng, V. Lesk, C. De Jager, 'Cognitive assessment: Discrimination of impairment and detection of decline in Alzheimer's disease and mild cognitive impairment.' In B. Klimova, M. Valis, K. Kuca, 'Potential of 
mobile technologies and applications in the detection of mild cognitive impairment among older generation groups.', Social Work in Health Care, Vol.56(7), p.p. 588-599, Aug. 2017. https://doi.org/10.1016/j.jalz.2008.05.1676

[56] K. Onoda, and S. Yamaguchi, 'Revision of the Cognitive Assessment for Dementia, iPad version (CADi2).' PloS One, Vol. 9(10), e109931, Oct 2014. https://doi.org/10.1371/journal.pone.0109931

[57] L. Scanlon, E. O'Shea, R. O' Caoimh, S. Timmons, 'Usability and Validity of a Battery of Computerized Cognitive Screening Tests for Detecting Cognitive Impairment.', Gerontology, Vol.62(2), p.p. 247-252, Jun 2015. https://doi.org/10.1159/000433432.

[58] United Nations, Department of Economic and Social Affairs, Population Division (2019). World Population Prospects 2019: Highlights. ST/ESA/SER.A/423. https://doi.org/10. 18356/f9a66f86-en

\section{Authors}

Andreas Solias is a social worker at the Municipality of Ilion, Attica, Greece since 2006. He received the Diploma of Social Worker from Technological Educational Institute of Athens (1992) and his master's degree in Promotion of Mental Health - Prevention of Psychiatric Disorders, from the Medical School of National and Kapodestrian University of Athens (2012). He has been a PhD candidate of the Medical School of Aristotle University of Thessaloniki (2016-2019). Also, he has been Deputy Secretary-General at the Hellenic Red Cross $(2010$ - 2013). He has worked at Therapeutic Communities for drug addicts (1988-1994) and in the Hellenic Red Cross (1995-2006). His main research interests include a) mild and major neurocognitive disorders, b) the development of computerized screening tests c) Electroencephalograms and eye-tracking signals d) anxiety and affective disorders in older adults. He also interested in research and clinical work with drug addicts, homeless and unemployed people. His involvement in these areas has led to the author in 10 papers in international or national journals and conferences.

Agisilaos Chaldogeridis is a $\mathrm{PhD}$ candidate in School of Informatics of Aristotle University of Thessaloniki (AUTH). He received his bachelor's degree from School of Informatics of Aristotle University of Thessaloniki in 2010. In 2012, he received his master's degree in "Information and Communication Technologies in Education" from School of Informatics of Aristotle University of Thessaloniki too. Since 2013 he is a $\mathrm{PhD}$ candidate at the aforementioned department and his main domain of research is "Cognitive Training supported by Communication and Information Technologies". While pursuing his doctoral degree, he is participating as a research associate in several research projects including all kinds of software development and IT support. Since 2016, he works as software engineer and developer in private sector.

Areti Batzikosta is a Psychologist, BSc, MSc, $\mathrm{PhD}$ candidate, senior researcher on issues of Mild Cognitive Impairment and Sleep Disorders. She received her Diploma degree from the School of Psychology of the Aristotle University of Thessaloniki in 2011. Also received her MSc degree in "Sleep Medicine" from the School of Medicine, of the Democritus University of Thrace. Soon after she started her doctoral thesis and since then, she works systematically on issues of Mild Cognitive Impairment and Sleep 
Physiology. She also participates as a senior researcher in groups in the field of neurocognitive disorders.

Magdalini Tsolaki was born in Thessaloniki, Greece and studied Medicine (19721978) and Theology (1981-1985) at the Aristotle University of Thessaloniki (AUTh). Afterwards, she initiated her collaboration with AUTh in 1982 as scientific collaborator and a year later received her specialty as a Neuropsychiatrist. She has been a Professor of Neurology since 2010, and currently, she is the Head of the 1st University Department of Neurology (since 2017) where she is still committed to her clinical, educational and research work. In 2004, she was invited as a visiting professor at the Boston University, Massachusetts, USA. She has formed the Greek Alzheimer Association in 1995 and the Greek Federation of Alzheimer's Disease (AD) in 2007, in which she is up to today the Chair, while also being the scientific director of two Public Dementia Units (founded by her in 2007 and 2009) for outpatients. The last two years she was invited to join as a member of the Greek National Observatory for Dementia. As a chair of the Greek Federation of Alzheimer's Disease, she has given more than 516 lectures throughout Greece. She has participated in more than 40 funded research programmes and 31 funded clinical trials. In total, she has received 70 awards. Dr Tsolaki has been the main author of many Books (53), many abstracts in Greek (531) and International (466) Conferences, she is the first author or co-author in many Papers in Greek (288) and International Journals (464 - 376 in PubMed), (h-index=67 and more than 20.000 Citations in Google Scholar). She has participated as invited speaker in 100 meetingsconferences. Furthermore, she has been a reviewer for Conferences and Journals (430) and has organised 27 national and 5 international conferences on AD. Finally, she was one of the three or seven advisory members for 30 doctoral theses-completed. Pr. Tsolaki is married with four children and ten grandchildren.

Article submitted 2020-04-05. Resubmitted 2020-05-17. Final acceptance 2020-05-24. Final version published as submitted by the authors. 